\title{
TUNNELING OF DIRAC PARTICLES FROM PHANTOM REISSNER NORDSTROM ADS BLACK HOLE
}

\author{
Gohar, H. \\ Department of Mathematics, Karakoram International University Gilgit, Pakistan
}

Received 2013-10-19; Revised 2013-11-09; Accepted 2013-11-27

\begin{abstract}
We study the Hawking radiation of Dirac particles from Phantom Reissner Nordstrom ADS black hole by tunneling method. We use charge relativistic Dirac equation to study the emission of such particles. To solve Dirac equation we use WKB approximation and find the tunneling probability of outgoing particles. Finally we find the Hawking temperature for such type of black holes.
\end{abstract}

Keywords: ADS Black Holes, Quantum Tunneling, Hawking Radiation, Dirac Particles

\section{INTRODUCITON}

Particles and anti particles are created permanently at the event horizon of the black hole due to the high gravitational fields and vacuum fluctuations. Stephan Hawking theoretically showed that one of the particle can go out of the black hole by calculating the Bogoliubov transformation between the initial and final states of ingoing and outgoing radiation (Hawking, 1974; 1975; Birrel and Davies, 1982). In this way Wick rotation Method (Gibbons and Hawking, 1977a; 1977b), Anomaly method (Iso et al., 2006) and methods of dimensional reduction (Umetsu, 2010) has been widely used by different authors to investigate the Hawking radiations from different black holes. Similarly Hawking radiation as quantum tunneling effect at event horizons of the black holes is discussed by different authors and quantum tunneling method (Parikh and Wilczek, 2000; Padmanabhan, 2004; Srinivasan and Padmanabhan, 1999; Shankaranarayanan et al., 2002; Kraus and Wilczek, 2009; 1995; Kerner and Mann, 2006; 2007; 2008; Rehman and Saifullah, 2011; Gillani and Saifullah, 2011; Ahmed and Saifullah, 2011; Gohar and Saifullah 2012a, 2012b, 2013; Jan and Gohar, 2013) is robust method to discuss these radiation. We can apply this method to almost all type of black holes in different gravity theories from higher dimensions to lower dimensions (Ejaz et al., 2013; Vagenas, 2002; Medved and Vagenas, 2005; Matsuno and Umetsu, 2011; Kim,
2011; Hod, 2011; Wu and Peng, 2011). In this study we have used the tunneling method to discuss the emission of Dirac particles from Phantom Reissner Nordstrom ADS black hole and finally get the Hawking temperature for this black hole.

\section{PHANTOM REISSNER NORDSTROM ADS BLACK HOLE}

Phantom Reissner Nordstrom ADS black hole (RN ADS black hole) is a solution of Einstein field equations in the presence of cosmological constant. This solution arises due to coupling of a field of spin 1 with gravitational field. The field of spin 1 may be the usual Maxwell one, or with a contribution of negative energy density, called phantom. The action for this theory is given by (Jardim et al., 2012) Equation (1):

$S=\int d^{4} x \sqrt{-g}\left[R+2 \eta F_{\mu v} F^{\mu v}+2 \Lambda\right]$

where, the first part of the action is the Einstein Hilbert action, second is the coupling with phantom field of spin 1 for $\eta=-1$ or coupling with Maxwell field for $\eta=1$ and third term is coupling with cosmological constant, $\Lambda$. For $\Lambda>0$, It behaves as De Sitter (DS) and for $\Lambda<0$, It behaves as Anti De Sitter (ADS). $R$ is the Ricci scalar and $F_{\mu v}=A_{\mu, v}$ -.- $A_{v, \mu}$, where $A_{\mu}$ is the four potential. The line element for such type of black holes is given by Equation ( 2 and 3 ): 
$d s^{2}=-g(r) d t^{2}+[g(r)]^{-1} d r^{2}+r^{2}\left(d \theta^{2}+\sin ^{2} \theta d \phi^{2}\right)$

Where:

$g(r)=1-\frac{2 M}{r}-\frac{\Lambda}{3} r^{2}+\eta \frac{Q^{2}}{r^{2}}$

Here, $\mathrm{M}$ and $\mathrm{Q}$ are the mass and charge of the black hole. For $\eta=1$, the black hole is called RN ADS black hole and when for $\eta=-1$; it is called anti RN ADS black hole. The event horizon can be found by putting $\mathrm{g}(\mathrm{r})=0$ so we have two horizon, $\mathrm{r}_{+}$and $\mathrm{r}_{\text {. }}$. For RN ADS; we have $0<r_{-}<r_{+}$and for anti RN ADS; we have $r_{-}<0<r_{+}$. For anti RN ADS; we have the event horizon given by (Jardim et al., 2012) Equation (4):

$\mathrm{r}_{+}=\frac{1}{2}\left(\sqrt{\mathrm{x}}+\sqrt{\frac{6}{\Lambda}-\mathrm{x}-\frac{12 \mathrm{M}}{\Lambda \sqrt{\mathrm{x}}}}\right)$

Where Equation (5 to 8):

$\mathrm{x}=\mathrm{A}+\mathrm{B}+\frac{2}{\Lambda}$

$\mathrm{A}=-\sqrt[3]{\frac{2}{\mathrm{y}}}\left(\frac{1-4 \Lambda \eta \mathrm{Q}^{2}}{\Lambda}\right)$

$\mathrm{B}=-\sqrt[3]{\frac{\mathrm{y}}{32}}\left(\frac{3}{\Lambda}\right)$

$y=2-36 \Lambda M^{2}+24 \eta \Lambda Q^{2}+$

$\left(\left(2-36 \Lambda \mathrm{M}^{2}+24 \eta \Lambda \mathrm{Q}^{2}\right)^{2}-4\left(1-4 \eta \Lambda \mathrm{Q}^{2}\right)^{3}\right)^{\frac{1}{2}}$

The mass of the black hole is given by Equation (9):

$\mathrm{M}=\frac{\mathrm{r}_{+}}{2}\left(1-\frac{\Lambda}{3} \mathrm{r}_{+}{ }^{2}+\frac{\eta \mathrm{Q}^{2}}{\mathrm{r}_{+}^{2}}\right)$

\section{QUANTUM TUNNELING}

To investigate the Hawking radiation of Dirac particles or spin half fermions from the black hole, we use the relativistic charged Dirac equation with Dirac field $\psi=\psi(\mathrm{t}, \mathrm{r}, \theta, \phi)$ given by Equation (10):

$\mathrm{i} \hbar \gamma^{\mu}\left(\partial_{\mu}+\Omega_{\mu}-\mathrm{iqA}_{\mu}\right) \psi+\mathrm{m} \psi=0$ where, $\mathrm{m}$ and $\mathrm{q}$ are the mass and charge of the particle. $A_{\mu}$ is the electromagnetic four potential and other parameters are defined as follow Equation (11 and 12):

$\Omega_{\mu}=\frac{i}{2} \Gamma_{\mu}^{\alpha \beta} \sum_{\alpha \beta}$

$\sum_{\alpha \beta}=\frac{i}{4}\left[\gamma^{\alpha}, \gamma^{\beta}\right]$

Here, $\gamma^{\mu}$ matrices satisfy $\left[\gamma^{\mu}, \gamma^{v}\right]=2 \mathrm{~g}^{\mu v} \times \mathrm{I}$, where $\mathrm{I}$ is the identity matrix. We use the following $\gamma^{\mu}$ matrices to study the tunneling of Dirac particles from the horizons of anti RN ADS black hole Equation (13 and 14):

$$
\begin{aligned}
& \gamma^{\mathrm{t}}=\frac{1}{\sqrt{\mathrm{g}(\mathrm{r})}}\left(\begin{array}{cc}
\mathrm{i} & 0 \\
0 & -\mathrm{i}
\end{array}\right), \gamma^{\mathrm{r}}=\sqrt{\mathrm{g}(\mathrm{r})}\left(\begin{array}{cc}
0 & \sigma^{3} \\
\sigma^{3} & 0
\end{array}\right) \\
& \gamma \theta=\frac{1}{\mathrm{r}}\left(\begin{array}{cc}
0 & \sigma^{1} \\
\sigma^{1} & 0
\end{array}\right), \quad \gamma^{\phi}=\frac{1}{\mathrm{r} \sin \theta}\left(\begin{array}{cc}
0 & \sigma^{2} \\
\sigma^{2} & 0
\end{array}\right)
\end{aligned}
$$

Pauli sigma, $\sigma^{\mathrm{i}}$, matrices are given by Equation (15):

$$
\sigma^{1}=\left(\begin{array}{ll}
0 & 1 \\
1 & 0
\end{array}\right), \sigma^{2}=\left(\begin{array}{cc}
0 & -i \\
i & 0
\end{array}\right), \sigma^{3}=\left(\begin{array}{cc}
1 & 0 \\
0 & -1
\end{array}\right)
$$

For a Dirac particle, the wave function $\psi$ has two spin states namely spin up and spin down so we can take the following ansatz for this wave function $\psi$ Equation (17):

$\psi_{\uparrow}=\left(\begin{array}{c}\mathrm{A}(\mathrm{t}, \mathrm{r}, \theta, \phi) \\ 0 \\ \mathrm{~B}(\mathrm{t}, \mathrm{r}, \theta, \phi) \\ 0\end{array}\right) \exp \left(\frac{\mathrm{i}}{\hbar} \mathrm{I}_{\uparrow}(\mathrm{t}, \mathrm{r}, \theta, \phi)\right)$

$\psi_{\downarrow}=\left(\begin{array}{c}0 \\ \mathrm{C}(\mathrm{t}, \mathrm{r}, \theta, \phi) \\ 0 \\ \mathrm{D}(\mathrm{t}, \mathrm{r}, \theta, \phi)\end{array}\right) \exp \left(\frac{\mathrm{i}}{\hbar} \mathrm{I}_{\downarrow}(\mathrm{t}, \mathrm{r}, \theta, \phi)\right)$

Here $\mathrm{I} \uparrow$ is the action for classical outgoing trajectory. Here we deal with only spin up case and for spin down case the calculations are same with signature changes. Put Equation (16) in Equation (10) and using lowest WKB approximation, we get the following four equations in leading order of $\hbar$ Equation (18 to 21): 


$$
\begin{aligned}
& -\left(\frac{\mathrm{iA}\left(\partial_{\mathrm{t}} \mathrm{I}_{\uparrow}-\mathrm{qA} \mathrm{A}_{\mathrm{t}}\right)}{\sqrt{\mathrm{g}(\mathrm{r})}}+\mathrm{B} \sqrt{\mathrm{g}(\mathrm{r})} \partial_{\mathrm{r}} \mathrm{I}_{\uparrow}\right)+\mathrm{mA}=0 \\
& \left(\frac{\mathrm{iB}\left(\partial_{\mathrm{t}} \mathrm{I}_{\uparrow}-\mathrm{qA} \mathrm{A}_{\mathrm{t}}\right)}{\sqrt{\mathrm{g}(\mathrm{r})}}-\mathrm{A} \sqrt{\mathrm{g}(\mathrm{r})} \partial_{\mathrm{r}} \mathrm{I}_{\uparrow}\right)+\mathrm{mB}=0 \\
& \frac{-\mathrm{B}}{\mathrm{r}}\left(\partial_{\theta} \mathrm{I}_{\uparrow}+\frac{\mathrm{i}}{\sin \theta} \partial_{\phi} \mathrm{I}_{\uparrow}\right)=0 \\
& \frac{-\mathrm{A}}{\mathrm{r}}\left(\partial_{\theta} \mathrm{I}_{\uparrow}+\frac{\mathrm{i}}{\sin \theta} \partial_{\phi} \mathrm{I}_{\uparrow}\right)=0
\end{aligned}
$$

If we look at the symmetries of the space time, $\partial_{\mathrm{t}}$ and $\partial_{\phi}$ are Killing fields in the respective direction and we are only dealing with radial trajectories, so we choose ansatz of the form:

$$
\mathrm{I}_{\uparrow}=-\mathrm{Et}+\mathrm{W}(\mathrm{r}, \theta)+\mathrm{J} \phi
$$

$\mathrm{E}$ is the energy of the particle and $\mathrm{J}$ is the corresponding angular momentum of the particle. Putting Equation (22) in above equations for $\theta=\theta_{0}$ and considering only the radial trajectory so by assuming $\mathbf{J}=$ 0 , we get the following two equations:

$$
\begin{aligned}
& -\left(\frac{i A\left(-E-q A_{t}\right)}{\sqrt{g(r)}}+B \sqrt{g(r)} \partial_{r} w\right)+m A=0 \\
& \left(\frac{i B\left(-E-q A_{t}\right)}{\sqrt{g(r)}}+A \sqrt{g(r)} \partial_{r} w\right)+m B=0
\end{aligned}
$$

If we put mass of the particle, $\mathrm{m}=0$, we have two solutions for Equation (23) and (24). For Equation (26):

$$
\begin{aligned}
& A=-i B \text {, we have } \partial_{r} W(r)=\partial_{r} W_{+}=\frac{E+q A_{t}}{g(r)} \\
& A=i B \text {, we have } \partial_{r} W(r)=\partial_{r} W-=-\frac{\left(E+q A_{t}\right)}{g(r)}
\end{aligned}
$$

Plus and minus signs correspond to the outgoing and incoming particles. Here in Equation (25), we have simple pole at $r=r_{+}$so we use reside theory for semi circle and we get:

$$
\mathrm{W}_{ \pm}= \pm \mathrm{i} \pi \frac{\mathrm{E}+\mathrm{qA} \mathrm{A}_{\mathrm{t}}}{\partial_{\mathrm{r}} \mathrm{g}\left(\mathrm{r}_{+}\right)}
$$

The overall tunneling probability is given by taking ratio of outgoing and in-coming rates to get the correct tunneling rate is given by (Srinivasan and Padmanabhan, 1999; Shankaranarayanan et al., 2002) Equation (28 and 27):

$$
\begin{aligned}
& \Gamma=\frac{\mathrm{P}_{\text {out }}}{\mathrm{P}_{\text {in }}}, \text { where } \\
& \mathrm{P}_{\text {out }} \propto \exp (-2 \operatorname{Im}(\mathrm{I}))=\exp \left(-2 \operatorname{Im}\left(\mathrm{W}_{+}\right)\right) \\
& \mathrm{P}_{\text {in }} \propto \exp (-2 \operatorname{Im}(\mathrm{I}))=\exp \left(-2 \operatorname{Im}\left(\mathrm{W}_{-}\right)\right)
\end{aligned}
$$

We can write the overall tunneling probability as:

$\Gamma=\exp \left(-4 \operatorname{Im}\left(\mathrm{W}_{+}\right)\right)$

By putting the value from Equation (27), we get the tunneling probability for outgoing particles as Equation (31):

$$
\Gamma=\exp \left(-4 \pi \frac{E+q A_{t}}{\partial_{r} g\left(r_{+}\right)}\right)
$$

Or:

$$
\Gamma=\exp \left(\frac{-2 \pi\left(E+\frac{\mathrm{qQ}}{\mathrm{r}_{+}}\right)}{\frac{\mathrm{M}}{\mathrm{r}_{+}^{2}}-\frac{\Lambda}{2} \mathrm{r}_{+}-\eta \frac{\mathrm{Q}^{2}}{\mathrm{r}_{+}^{3}}}\right)
$$

From Equation (32) we can conclude that the tunneling probability does not depend upon the mass of the particle. Comparing Equation (32), with the Boltzmann factor, we get the Hawking temperature for this black hole and is given by:

$$
\mathrm{T}_{\mathrm{bh}}=\frac{1}{2 \pi}\left(\frac{\mathrm{M}}{\mathrm{r}_{+}^{2}}-\frac{\Lambda}{3} \mathrm{r}_{+}-\eta \frac{\mathrm{Q}^{2}}{\mathrm{r}_{+}^{3}}\right)
$$

For Massive particle, $m \neq 0$, we get:

$$
\left(\frac{A}{B}\right)^{2}=\frac{-i E+m \sqrt{g(r)}}{i E+m \sqrt{g(r)}}
$$


When we approach near the horizon then we have $\left(\frac{A}{B}\right)^{2}=-1$ and we get the Hawking temperature similar to massless case. This is because at event horizon massive particle behaves like massless particle for more detail (Kerner and Mann, 2008). We have also taken care of the temporal contribution to the imaginary part of the action so that for no value of the energy, charge and angular momentum of the particles will the tunneling probabilities be greater than 1 (Akhmedov et al., 2008; Akhmedova, 2008) and they will not violate unitarity.

If we look at thermodynamic relations for this black hole then mass of the black hole is given by putting $\mathrm{g}(\mathrm{r}+)$ $=0$ Equation (34):

$\mathrm{M}=\frac{\mathrm{r}_{+}}{2}\left(1-\frac{\Lambda}{3} \mathrm{r}_{+}^{2}+\frac{\eta \mathrm{Q}^{2}}{\mathrm{r}_{+}^{2}}\right)$

The entropy of the Black hole is given by Equation (35):

$$
\mathrm{S}=\frac{\mathrm{A}}{4}=\pi \mathrm{r}_{+}^{2}
$$

For the electric potential V Equation (36):

$$
\frac{\partial \mathrm{M}}{\partial \mathrm{Q}} \mid \mathrm{s}=\mathrm{V}=\frac{\eta \mathrm{Q}}{\mathrm{r}_{+}}
$$

Now we have:

$$
\left.\frac{\partial \mathrm{M}}{\partial \mathrm{S}}\right|_{\mathrm{Q}}=\mathrm{T}=\frac{1}{2 \pi}\left(\frac{\mathrm{M}}{\mathrm{r}_{+}^{2}}-\frac{\Lambda}{3} \mathrm{r}_{+}-\eta \frac{\mathrm{Q}^{2}}{\mathrm{r}_{+}^{3}}\right)
$$

Which is from the thermodynamic relations and Equation (37) is same as the Hawking temperature calculated from quantum tunneling method. After putting the value of $\mathrm{M}$, We have the Hawking temperature Equation (38):

$$
\mathrm{T}_{\mathrm{bh}}=\frac{1}{4 \pi \mathrm{r}+}\left(1-\Lambda \mathrm{r}_{+}^{2}-\eta \frac{\mathrm{Q}^{2}}{\mathrm{r}_{+}^{2}}\right)
$$

Which is consistent with previous literature (Jardim et al., 2012).

\section{CONCLUSION}

By ignoring the self gravity and back reaction of the Dirac particle, we have discussed quantum tunneling of Dirac particles from the event horizon of the phantom ADS black hole. For this purpose, we have used the relativistic Dirac Equation and found the tunneling probability of outgoing fermions and we found the Hawking temperature for ADS black holes. From Equation (32), we can say that the tunneling probability depends upon the charge of the particle but not on the mass of the particle. We have justified our results by writing the usual thermodynamic relations and which are same by using the tunneling method. Last but not least, one can also find the tunneling with back reaction from these black holes, which will have very interesting results.

\section{REFERENCES}

Ahmed, J. and K. Saifullah, 2011. Hawking radiation of Dirac particles from black strings. J. Cosmol. Astropart. Phys., 08: 011-011. DOI: 10.1088/14757516/2011/08/011

Akhmedov, E.T., T. Pilling and D. Singleton, 2008. Subtleties in the quasi-classical calculation of hawking radiation. Int. J. Mod. Phys. D., 17: 24532458. DOI: $10.1142 / \mathrm{S} 0218271808013947$

Akhmedova, V., T. Pilling, A. de Gill and D. Singleton, 2008. Temporal contribution to gravitational WKBlike calculations. Phys. Lett. B, 666: 269-271. DOI: 10.1016/j.physletb.2008.07.017

Birrel, N.D. and P.C.W. Davies, 1982. Quantum Fields in Curved Space. 1st Edn., Cambridge University Press, Cambridge, ISBN-10: 9780511622632. DOI: 10.1017/CBO9780511622632

Ejaz, A., H. Gohar, H. Lin, K. Saifullah and S.T. Yau, 2013. Quantum tunneling from three-dimensional black holes. Phys. Lett. B, 726: 827-833. DOI: 10.1016/j.physletb.2013.09.015

Gibbons, G.W. and S.W. Hawking, 1977a. Cosmological event horizons, ther-modynamics and particle creation. Phys. Rev., D, 15: 2738-2751. DOI: 10.1103/PhysRevD.15.2738

Gibbons, G.W. and S.W. Hawking, 1977b. Action integrals and partition functions in quantum gravity. Phys. Rev. D, 15: 2752-2756. DOI: 10.1103/PhysRevD.15.2752

Gillani, U.A. and K. Saifullah, 2011. Tunneling of Dirac particles from accelerating and rotating black holes. Phys. Lett. B, 699: 15-20. DOI: 10.1016/j.physletb.2011.03.051 
Gohar, H. and K. Saifullah 2012b. Scalar field radiation from dilatonic black holes. Gen. Relativ. Gravit. 44, 3163-3167. DOI: 10.1007/s10714-012-1449-x

Gohar, H. and K. Saifullah, 2012a. Emission of scalar particles from cylindrical black holes. Astrophys. Space Sci., 343: 181-185. DOI: 10.1007/s10509012-1255-7

Gohar, H. and K. Saifullah, 2013. Quantum tunneling from scalar fields in rotating black strings. Astropart. Phys. 48, 82-85. DOI: 10.1016/j.astropartphys.2013.07.004

Hawking, S.W., 1974. Black hole explosions? Nature, 248: 30-31. DOI: $10.1038 / 248030 \mathrm{a} 0$

Hawking, S.W., 1975. Particle creation by black holes. Commun. Math. Phys., 43: 199-220. DOI: 10.1007/BF02345020

Hod, S., 2011. Bulk emission by higher-dimensional black holes: Almost perfect blackbody radiation. Class. Quant. Grav., 28: 105016. DOI: 10.1088/0264-9381/28/10/105016

Iso, S., H. Umetsu and F. Wilczek, 2006. Anomalies, Hawking radiations and regularity in rotating black holes. Phys. Rev. D, 74: 044017-044026. DOI: 10.1103/PhysRevD.74.044017

Jan, K. and H. Gohar, 2013. Hawking radiation of scalars from accelerating and rotating black holes with NUT parameter. Astrophys. Space Sci. DOI: 10.1007/s10509-013-1704-y

Jardim, D.F., M.E. Rodrigues and S.J.M. Houndjo, 2012. Thermodynamics of phantom Reissner-NordstromAdS black hole. Eur. Phys. J. Plus, 127: 123-123. DOI: 10.1140/epjp/i2012-12123-X

Kerner, R. and R.B. Mann, 2006. Tunnelling, temperature and Taub-Nut black holes. Phys. Rev. D, 73: 104010-104020. DOI: 10.1103/PhysRevD.73.104010

Kerner, R. and R.B. Mann, 2007. Tunnelling from Godel black holes. Phys. Rev. D, 75: 084022-084030. DOI: 10.1103/PhysRevD.75.084022

Kerner, R. and R.B. Mann, 2008. Charged Fermions tunnelling from Kerr-Newman black holes. Phys. Lett. B, 665: 277-283. DOI: 10.1016/j.physletb.2008.06.012

Kim, H., 2011. Hawking radiation as tunneling from charged black holes in 0A string theory, Phys. Lett. B, 703: 94-99. DOI: 10.1016/j.physletb.2011.07.053

Kraus, P. and F. Wilczek, 1995. Effect of selfinteraction on charged black hole radiance. Nucl. Phys. B, 437: 231-242. DOI: 10.1016/05503213(94)00588-6
Kraus, P. and F. Wilczek, 2009. A simple stationary line element for the Schwarzschild geometry and some applications.

Matsuno, K. and K. Umetsu, 2011. Hawking radiation as tunneling from squashed Kaluza-Klein black hole. Phys. Rev. D, 83: 064016-064022. DOI: 10.1103/PhysRevD.83.064016

Medved, A.J.M. and E.C. Vagenas, 2005. On hawking radiation as tunneling with back-reaction. Mod. Phys. Lett., 20: 2449-2454. DOI: $10.1142 / \mathrm{S} 021773230501861 \mathrm{X}$

Padmanabhan, T., 2004. Entropy of horizons, complex paths and quantum tunnelling. Mod. Phys. Lett. A, 19: 2637-2643. DOI: 10.1142/S0217732304015257

Parikh, M.K. and F. Wilczek, 2000. Hawking radiation as tunneling. Phys. Rev. Lett., 85: 5042-5045. DOI: 10.1103/PhysRevLett.85.5042

Rehman, M. and K. Saifullah, 2011. Charged fermions tunneling from accelerating and rotating black holes. J. Cosmol. Astropart. Phys. DOI: 10.1088/14757516/2011/03/001

Shankaranarayanan, S., T. Padmanabhan and K. Srinivasan, 2002. Hawking radiation in different coordinate settings: Complex paths approach. Class. Quantum Gravity, 19: 2671-2671. DOI: 10.1088/0264-9381/19/10/310

Srinivasan, K. and T. Padmanabhan, 1999. Particle production and complex path analysis. Phys. Rev. D, 60: 24007-24026. DOI: 10.1103/PhysRevD.60.024007

Umetsu, K., 2010. Tunneling mechanism in KerrNewman black hole and dimensional reduction near the horizon. Phys. Lett. B, 692: 61-63. DOI: 10.1016/j.physletb.2010.07.019

Vagenas, E.C., 2002. Complex paths and covariance of hawking radiation in 2D stringy black holes. Nuovo. Cim. B, 117: 899-908.

Wu, S.Q. and J.J. Peng, 2011. Thermodynamics and Hawking radiation of five-dimensional rotating charged Gödel black holes. Phys. Rev. D, 83: 044028-044037.

DOI: 10.1103/PhysRevD.83.044028 